\section{Die Zürcher Schule für aufgeklärte Nachhaltigkeit}

Transdisziplinarität sucht mögliche Lösungen für komplexe gesellschaftliche Probleme. Dafür bricht sie disziplinäre Engsicht auf und bringt außerwissenschaftliche Perspektiven ein. Sie übt Wissenskultur in Forschung und Praxis, die in der Nachhaltigkeitswissenschaft

Uwe Schneidewind, Otto Smrekar einen Vorreiter gefunden hat: die

environmental literacy, die uns vom

Wissen zum Entscheiden leitet.

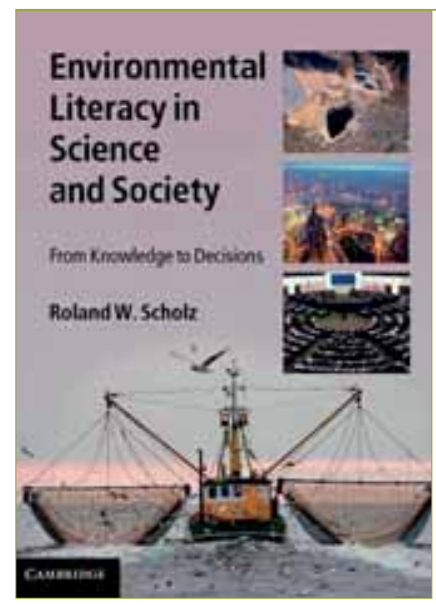

Scholz, R. W. 2011. Environmental literacy in science and society. From knowledge to decisions. New York: Cambridge University Press. 656 pp., 52.99 EUR, ISBN 978-0521-18333-8 eit ungefähr 20 Jahren wird über eine $\checkmark$ transdisziplinäre Nachhaltigkeitswissenschaft als neuen Forschungsmodus diskutiert, über ihre Konzeptualisierung, adäquate methodische Standards und die Anerkennung durch institutionelle Etablierung. In dieser Zeit war der Lehrstuhl für Natural and Social Science Interface am Institut für Umweltentscheidungen der ETH Zürich eines der Zentren bei der Entwicklung der transdisziplinären Nachhaltigkeitswissenschaft. Gegründet 1993 mit der Berufung von Roland Scholz, sind in Zürich seither konzeptionelle Grundlagen und insbesondere die transdisziplinäre Fallstudienmethodik entwickelt worden. Rund zehn Professuren in verschiedenen Disziplinen an Universitäten weltweit sind bisher aus dieser Schule hervorgegangen.

Mit Environmental Literacy in Science and Society - From Knowledge to Decisions erhebt Roland Scholz den Anspruch, ein umfassendes source book vorzulegen: In 20 Kapiteln zeigt der Autor die grundlegenden disziplinären, inter- und transdisziplinären

\footnotetext{
Kontakt: Prof. Dr. Uwe Schneidewind | Wuppertal Institut für Klima, Umwelt, Energie $\mathrm{GmbH}$ | Wuppertal | Deutschland | E-Mail: uwe.schneidewind@wupperinst.org

Dr. Otto Smrekar | Mase (Mont-Noble) | Schweiz | E-Mail: otto.smrekar@unibas.ch

(C) 2012 U. Schneidewind, O. Smrekar; licensee oekom verlag. This is an article distributed under the terms

of the Creative Commons Attribution License (http://creativecommons.org/licenses/by/3.0), which permits unrestricted use, distribution, and reproduction in any medium, provided the original work is properly cited.
}

Züge einer Nachhaltigkeitswissenschaft auf und entfaltet einen Methodenkanon zur Erforschung von Mensch-Umwelt-Systemen. Zum Quellenwerk wird das Buch durch seine konzeptionelle Klarheit sowie durch die didaktische Aufbereitung: mit Leserführung, Schlüsselfragen und -botschaften am Anfang und Ende jedes Kapitels, mit ausgearbeiteten Fallbeispielen und Illustrationen aus der Zürcher Praxis sowie mit Glossar, Index und 74-seitigem Literaturverzeichnis.

All dies dient dazu, die Leserinnen und Leser auf eine Reise in die transdisziplinäre Nachhaltigkeitswissenschaft mitzunehmen - geführt von einem Autor, der sich in jedem Winkel dieses Terrains zu Hause fühlt.

\section{Interaktive Mensch-Umwelt-Systeme als Ausgangspunkt}

Den Rahmen für Scholz' Analyse und Synthese bilden human-environment systems (HES). Dabei spannt das Buch den Anspruch von Anfang an weit und eröffnet so seine grundlegende Perspektive: human systems umfassen Individuen, Gruppen, Organisationen, Institutionen und die Gesellschaft als Ganzes. Eine Nachhaltigkeitswissenschaft, die environmental und human systems und vornehmlich die komplexe Interaktion beider Systeme verstehen, begreifen und erklären will, muss auf einem breiten disziplinären Fundament aufsetzen und darauf aufbauend die Konzepte und Methoden zu einer adäquaten Integration bereitstellen.
Somit wird das Buch gleichzeitig zu einem Vademecum der hier im Vordergrund stehenden Disziplinen von Biologie, Psychologie, Soziologie, Ökonomie bis industrial ecology (jeder davon ist ein eigenes Kapitel gewidmet). Scholz zeigt auf, was diese Disziplinen zu Mensch-Umwelt-Systeminteraktionen zu sagen haben, wo ihre Grenzen und Potenziale liegen. Allen Kapiteln gemein ist, dass der Autor sie mit führenden Vertretern der jeweiligen Fächer diskutiert hat und der Leserschaft so profunde Einblicke in die aktuellen Entwicklungslinien dieser Disziplinen geboten werden.

\section{Disziplinierte Interdisziplinarität in transdisziplinären Prozessen}

Mit der disziplinären Fundierung schafft Scholz gleich auch die Basis für eine der Schlüsselformeln seines Buchs. Dass die Untersuchung von Mensch-Umwelt-Systemen auf eine „disziplinierte Interdisziplinarität in transdisziplinären Prozessen“ (S.XVII) angewiesen ist, verdeutlichen das Zusammenspiel und die Komplementarität disziplinärer, interdisziplinärer und transdisziplinärer Forschung.

Wer Mensch-Umwelt-Systeme verstehen will, benötigt durchaus den disziplinären Zugang zu den einzelnen Systemen: Disziplinäre Konzepte und Modelle bereiten die Grundlage für „disziplinierte Interdisziplinarität“ im Austausch zwischen den Disziplinen und in der Erklärung von Wechselbeziehungen zwischen Mensch und Umwelt. Doch erst durch transdiszi- 
plinäre Prozesse, also Prozesse, in die das - oft implizite - Wissen gesellschaftlicher Akteure einbezogen wird, entsteht „sozial robustes Wissen“. Und dann können Lernprozesse in Wissenschaft und Gesellschaft in Gang gesetzt werden, die eine nachhaltigkeitsorientierte Weiterentwicklung von Mensch-Umwelt-Systemen ermöglichen. Wissensintegration wird damit zu einem Schlüsselkonzept transdisziplinärer Nachhaltigkeitswissenschaft. Dazu passt das Losungswort „Koevolution“ (Schellnhuber 2001).

Die Qualität des Werks zeigt sich darin, dass es nicht auf der abstrakten Ebene stehenbleibt. Vielmehr fließen hier 20 Jahre Erfahrung in transdisziplinären Prozessen ein, merkbar auf verschiedenen Ebenen:

- bei der Darlegung des Methodenkanons einer integrierten Systemmodellierung - insbesondere ist hier die Systematisierung unterschiedlicher Modellansätze im Hinblick auf ihre Erklärungsreichweiten hilfreich;

- beim erläuternden Betrachten von vier der über 20 großen Fallstudien, was die Forschung und die Prinzipien von transdisziplinären Prozessen plastisch werden lässt;

- bei der Darstellung der Postulate und des darauf gestützten HES frameworks als verdichteter Bezugsrahmen des eigenen Forschungsansatzes.

Im Kapitel Comparing the HES Framework with Alternative Approaches setzt Scholz seinen Ansatz zu anderen integrierenden Konzepten wie der Resilienzforschung, dem des sozialen Metabolismus und dem für Transition-Management in Bezug. Die Leser(innen) erhalten dadurch nicht nur eine prägnante Einführung in alternative Ansätze, sondern erfahren zudem, welch hilfreiches Navigationssystem die environmental literacy bietet, um die Reichweite der diversen Ansätze beurteilen zu können.

\section{Literacy als Kernkompetenz}

Dies führt zur programmatischen Titelwahl Environmental Literacy, die Scholz als „the ability to read and utilize environmental information appropriately, to anticipate rebound effects, and to adapt to changes in environmental resources and systems, and their dynamics" versteht (S. 540 f.) - die epochale Herausforderung des 21. Jahrhunderts.

Der Blick auf den aktuellen Nachhaltigkeitsdiskurs zeigt, dass wir erst über einzelne Bausteine einer solchen Kompetenz einer sustainability science. In Deutschland hat gerade der Wissenschaftliche Beirat der Bundesregierung Globale Umweltveränderungen (WBGU) eine massive Reorientierung im Wissenschaftssystem hin zu einer „transformativen Wissenschaft“ ge-

\section{Die Idee der gemeinsamen Lernprozesse zwischen Wissenschaft und Gesellschaft auf Augenhöhe bedeutet eine Chance zur Entwicklung nachhaltiger Gesellschaften.}

in Wissenschaft und Gesellschaft verfügen - immerhin hoffnungsvolle Inseln in der naturwissenschaftlichen und technologischen Bildungslandschaft. Unsere sozialwissenschaftliche literacy ist dagegen weniger ausgebildet, eine interdisziplinäre für die Natur-, Geistes- und Sozialwissenschaften zusammen ist der Ausnahmefall und die von transdisziplinären Prozessen kaum vorhanden. Die Idee der gemeinsamen Lernprozesse zwischen Wissenschaft und Gesellschaft auf Augenhöhe bedeutet eine Chance zur Entwicklung nachhaltiger Gesellschaften in näherer Zukunft.

An einem Knotenpunkt der Wissenschaftskulturen erarbeitet, soll das Werk den Meister loben: Environmental Literacy in Science and Society, exzeptionell konzipiert und ausgestaltet, lebt von seinem $\mathrm{Au}$ tor und Spiritus rector einer Schar von Kolleg(inn)en und Weggefährten, die ihn auf der Wegsuche from knowledge to decisions begleiten. Diese auf Orientierung bedachte Zusammenschau braucht Facettenaugen, die mit großem Blickfeld und hoher zeitlicher Auflösung komplexe, interdependente Systeme und gekoppelte Prozesse darin erfassen können. Der Wissenschaft kommt somit weit mehr als eine Beobachterrolle, nämlich auch eine Katalysatorfunktion zu.

Katalysator für die Transdisziplinaritätsdebatte auf europäischer Ebene

Durch seinen Kompendiumscharakter unterstützt das Buch die europäische und vor allem deutsche Debatte über die Zukunft fordert, um den Herausforderungen der anstehenden „großen Transformation“ $\mathrm{zu}$ nachhaltigen Gesellschaften gerecht $\mathrm{zu}$ werden (WBGU 2011). Die environmental literacy liefert die Blaupause für eine solche transformative Wissenschaft und zeigt, auf welch hohem konzeptionellen und methodischen Niveau heute geforscht wird. Das wird es auf nationaler und europäischer Ebene erleichtern, Maßnahmen zur institutionellen Durchsetzung voranzutreiben - wie sie aktuell zum Beispiel auf EUEbene im RD4SD-Prozess (Research and Development for Sustainable Development) diskutiert werden. Die Vision RD4SD versteht sich als Koordinations- und Supportaktion, die innerhalb des 7. Forschungsrahmenprogramms der Europäischen Kommission finanziell gefördert wird.

Ein robustes Netzwerk transdisziplinärer Laboratorien braucht den Rückhalt in Institutionen und den entschlossenen Auftrag aus der Politik. Die „Zürcher Mission“ empfiehlt sich als Drehkreuz zum Umdenken und Umsteigen. An einem operating manual for spaceship Earth wird noch lange zu laborieren sein. Aber das source book aus der Zürcher Schule für aufgeklärte Nachhaltigkeit vermittelt bereits enlightening experience and expertise.

\section{Literatur}

Schellnhuber, H.-J. 2001. Die Koevolution von Natur, Gesellschaft und Wissenschaft - Eine Dreiecksbeziehung wird kritisch. GAIA 10/4: 258-262.

WBGU (Wissenschaftlicher Beirat der Bundesregierung Globale Umweltveränderungen). 2011. Welt im Wandel. Gesellschaftsvertrag für eine Große Transformation. Berlin: WBGU. 